\title{
ON-ORBIT SPACECRAFT SERVICING AN ELEMENT IN THE EVOLUTION OF SPACE ROBOTICS APPLICATIONS
}

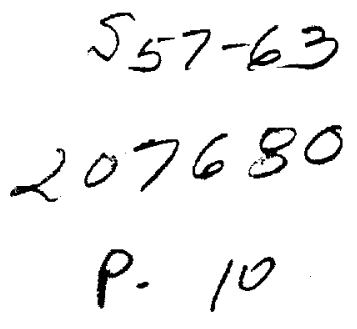

\author{
Senior Engineer, Advanced Systems \\ Spar Aerospace Ltd. \\ Advanced Technology Systems Group \\ 9445 Airport Road \\ Brampton, Ontario \\ Canada L6S 4J3
}

Carl J. Anders

Office: (905) 790-2800 FAX: (905) 790-4400

\author{
Claude H. Roy \\ Senior Marketing Specialist, Space Servicing \\ Spar Aerospace Ltd. \\ Advanced Technology Systems Group \\ 9445 Airport Road \\ Brampton, Ontario \\ Canada L6S 4J3 \\ Office: (905) 790-2800 FAX: (905) 790-4452
}

\begin{abstract}
This paper addresses the renewed interest in on-orbit spacecraft servicing (OSS), and how it fits into the evolution of space applications for intelligent robots.
\end{abstract}

Investment in the development of space robotics and associated technologies is growing as nations recognize that it is a critical component of the exploration and commercial development of space. At the same time, changes in world conditions have generated a renewal of the interest in OSS. This is reflected in the level of activity in the U.S., Japan and Europe in the form of studies and technology demonstration programs. OSS is becoming widely accepted as an opportunity in the evolution of space robotics applications. Importantly, it is a feasible proposition with current technologies and the direction of ongoing research and development activities. Interest in OSS dates back more than two decades, and several programs have been initiated, but no operational system has come on line, arguably with the Shuttle as the exception.

With new opportunities arising, however, a fresh look at the feasibility of OSS is warranted. This involves the resolution of complex market, technical and political issues, through market studies, economic analyses, mission requirement definitions, trade studies, concept designs and technology 
demonstrations. System architectures for OSS are strongly dependent on target. spacecraft design and launch delivery systems. Performance and cost factors are currently forcing significant changes in these areas. This presents both challenges and opportunities in the provision of OSS services.

In conclusion, there is no question OSS will become a reality, but only when the technical feasibility is combined with either economic viability or political will. In the evolution of space robotics satellite servicing can become the next step towards its eventual role in support of planetary exploration and human beings' journey out into the universe.

\section{SPACE ROBOTICS}

\section{Past and Present}

The first space-based robotic arm was the Shuttle Remote Manipulator System (SRMS), better known as the Canadarm. Space mechanisms date back almost to the first excursions into orbit in the late 1950s, early 1960s, and the first primitive 'robotic elements' were surface samplers on the planetary probes. The U.S. moon rover and unmanned Soviet rover should also receive honourable mention. However, the first true multidegree-of-freedom robotic manipulator was the SRMS, launched on the Space Transportation System (Shuttle) in 1981. To this day it remains the only operational space robotic arm. (See Figure 1.)

A number of technology demonstrations in space robotics are proposed or have been performed, but the next major development in the field will be the deployment on the Space Station of the Mobile Servicing System (MSS), currently scheduled to begin operations in 1998. Elements of this system include the Space Station Remote Manipulator System (SSRMS) a derivative of the SRMS, and the Special Purpose Dexterous Manipulator (SPDM) - a pair of arms equipped with special end effector tools, and designed for greater dexterity than the SSRMS. The MSS also includes a Ground Segment. (See Figure 2.)

\section{Looking to the Future}

There is a general consensus that robotics will play a major role in the exploration of space frontiers in the next century. Roles envisaged include: the construction of orbiting platforms, for manned occupation, materials storage, scientific experimentation and manufacturing; the assembly of planetary and interstellar expedition spacecraft; the construction and maintenance of habitats, and the construction, maintenance and operation of mining and industrial facilities on the moon and planets. Although manned presence will be desirable and necessary in many instances, safety and cost considerations are factors in moving space robotics development away from local manned operation towards teleoperated systems. Remote operation removes the risk to human life and eliminates the need for costly life-support systems. Even if astronauts are at the work site, the use of teleoperation can free up their valuable time for other tasks. Even more significant is the shift in thinking 
towards fully or semi autonomous systems, prompted by technology developments in such areas as sensing, artificial intelligence and predictive systems. This enables smart space robotic systems to function with minimal support from space or groundbased human operators. The penalty with the use of autonomous over teleoperated systems is the added weight and cost of placing high powered computational capabilities onboard the servicing vehicle.

\section{Technology Issues}

The application of teleoperation and autonomous robotic systems is by no means limited to space. Terrestrial tasks in isolated locations or hazardous environments have very similar requirements. This overlap extends to the critical technologies. For example, the two major problems inherent in the teleoperation of space systems are bandwidth limitations and time delays in the transmission and relay of signals (latency). These are the same issues found in deep sea (untethered) robotics applications.

The bandwidth limitations impose constraints on the quantity of data that can be transmitted between the ground station and the space system. The operator and the supporting computer facilities may have to work with incomplete or low resolution information.

It is generally accepted that latency greater than 0.25 to 0.5 seconds make real-time control by a human operator difficult if not impossible, and may demand a 'move-and-wait' approach.
This can be an acceptable control strategy in non-critical tasks, but has a high level of risk in complex, fast moving or close proximity operations.

An alternative to resolving the bandwidth and latency restrictions associated with teleoperation is to employ onboard autonomous control of space systems. The challenge then becomes design and installation of controllers with sophisticated sensing and highly developed intelligence.

Realistically in the near and medium term such operations will still require supervision and optional override by a human operator to handle unforseen contingencies.

The trend towards remote teleoperation and local autonomy in the control of robotic systems matches the projected long term needs of space exploration, and in the near term it opens the door for satellite servicing.

\section{ON-ORBIT SATELLITE SERVICING}

On-Orbit Spacecraft Servicing (OSS) is simply the provision of space-based services to orbiting craft. The interest in spacecraft servicing with its substantial benefit potential has led to the performance of a number of studies, proposals and programs. Despite this activity, and the success of Shuttle based on-orbit robotic operations, an OSS system has yet to be implemented.

\section{A Brief History}

The possibility of servicing spacecraft in orbit has generated considerable interest 
in the space community for over 20 years. A primary objective in the development of the U.S. Space Shuttle was to reduce space program costs by replacing expendable launch vehicles (ELVs) with a fully reusable system capable of maintaining, refurbishing and upgrading payloads. The original Shuttle concept included a space tug for the purpose of transporting satellites to and from a Shuttle-achievable orbit. However, projected high development costs forced a descope of the Shuttle capabilities, and the resulting configuration (the one flying today) has significantly reduced servicing capabilities, and no means of accessing high altitude orbits or high inclination low earth orbits (LEOs).

Many LEO spacecraft are accessible to the Shuttle, and several missions included the rescue and repair of scientific and communications spacecraft. The Shuttle program has been highly successful from a technical viewpoint in demonstrating OSS possibilities despite the constraints on the scope of its market and the failure of generated revenue to cover the mission costs.

The Challenger accident in 1986 had a dramatic impact on Shuttle operations with repercussions felt throughout the space industry. A direct result on the Shuttle was a greatly reduced launch capacity and very stringent mission restrictions forcing a shift in spacecraft designs from Shuttle launched systems to ones compatible with ELVs. With concurrent advances in satellite miniaturization, smaller, less expensive, expendable platforms became more attractive. Through the late 1980 s only a few large, complex platforms such as the Hubble Space Telescope were specifically designed to be serviceable by the Shuttle.

Access to a wider range of orbits including geosynchronous (GEO) was still desirable and a number of studies were commissioned to address that. None led to operational systems. Often, with a preconceived system architecture in mind, they focused on a single specific market. These markets were not always well chosen, and the systems lacked the flexibility to adapt to market changes. The most recent examples were the Orbital Maneuvring Vehicle (OMV) program and the Satellite Servicing System (SSS).

\section{ANew Perspective}

The history of space servicing has been somewhat inauspicious, but there is more interest than ever before with initiatives underway in the U.S., Canada, Japan and Europe. These involve market studies, economic analyses, mission requirement definitions, trade studies, concept designs and technology demonstrations in preparation for the development and implementation of pay-for-service systems. This revival of interest reflects a changing market. The old views and perspectives on servicing markets may no longer be applicable, and new opportunities are arising. An example of this is the shift towards Smallsats, and the first major application - Low Earth Orbit (LEO) constellations of communications spacecraft.

The unprecedented number of recent launch failures has kept the satellite insurance rates in a constant state of flux. The launch vehicle industry, itself 
a critical parameter in the OSS equation, is in transition. Increased commercialization promises reduced launch costs and greater availability. These and other factors will impact both the servicing and serviced systems, and suggests that a fresh look at OSS is warranted.

\section{Services}

The traditional concept of OSS is spacecraft repair or refurbishment, but services such as refuelling to extend operating lives, and the transportation of 'dead' vehicles into graveyard orbits are becoming increasingly important.

The term service can refer to any operation performed by one vehicle (servicer) on another vehicle (target or object). The primary possibilities for OSS include inspection, mechanical intervention, and repair/refurbishment activities. Payload upgrades may be offered through Orbital Replacement Unit (ORU) changeout. Refueling can be an important service since fuel capacity is the primary life-limiting factor for on-orbit satellites. Orbit transfer is a possibility for injection of a spacecraft into its correct orbit after apogee motor failure, correction of a drifting spacecraft, or placement of a 'dead' spacecraft into a graveyard orbit. Forced reentry into the earth's atmosphere may be a disposal option in LEO. Spacecraft harvesting, placement and retrieval of experiments, recovery of data, and spacecraft reconfiguration are also potential OSS services. On-orbit construction of space structures can replace the need for humans to work on such physically demanding tasks in a hostile environment. A distinct (if less likely) application is that of space debris clean-up.

\section{System Architecture Trades}

The development and implementation of an optimum commercial system involves the resolution of complex market, technical and political issues. Interwoven with each of these are the many economic factors that ultimately determine whether spacecraft owners and operators are willing to pay for onorbit services. From another perspective, satellite design life is balanced against payload obsolescence. The costs of making spacecraft serviceable must be weighed against service vehicle capability, and external influences such as fluctuating insurance rates.

The subject of technically and economically viable architectures for OSS is complicated by the fact that there are several potential markets, each of which presents a set of mission architecture trades. A satellite market can be segmented according to satellite type or function, and orbit. A further distinction is customer type. For example, commercial, civil government and defense communication satellites can have very different service requirements. An OSS system capable of satisfying a particular market has a distinct set of requirements, but many possible configurations. Not only will market shifts have a significant effect on the system architecture, but also on the cost and availability of the technology and hardware for the OSS system itself.

OSS system architectures can be assembled by considering the service 
base, service vehicle, control type and resupply vehicle. The system can be ground-based (service vehicle launched on demand), or space-based (service vehicle resident in space). The service vehicle may be expendable (short mission), or reusable (multi-mission). The control can be ground-based, spacebased, or local autonomous. If replacement of consumables is required, the resupply vehicle may also be either expendable or reusable.

One of the simplest system architectures that can be envisaged is a small autonomous spacecraft capable of visiting satellites and performing a nonintrusive inspection. An extension of this could be to add some robotic capability for simple mechanical tasks. A much more complex concept is that of a multi-purpose vehicle with the ability to inspect, repair, refuel or transport a satellite, perhaps operating from a spacebased storage depot that is resupplied from the ground. Another consideration is that an OSS system does not have to be based in space. It is conceivable to propose a quick response ground-based service system that would be launched on demand at short notice, an approach that may be preferred as launch costs decrease. The possibilities are almost unlimited.

\section{QSS Viability}

The conditions necessary for OSS to become a reality are the maturity of the required technology in conjunction with either economic viability or political will. The level of technology development particularly in the key area of space robotics is suitable for the implementation of an OSS system. The other half of the equation is not so clear.

The revenue-generating potential of OSS has been demonstrated by NASA's Shuttle-based repair and recovery of satellites. A good example of this was the reboost of Intelsat VI into its correct orbit in May 1992. Intelsat saved $\$ 200$ million over the alternative option to manufacture and launch a replacement spacecraft and lose revenue during the accompanying delay, despite their recovery expenses of $\$ 147$ million. This revenue level is not sufficient to finance a Shuttle mission generally costing in excess of $\$ 500$ million, and NASA failed to recover its own costs. It seems reasonable though to assume that with an appropriate commercial OSS system in place under such circumstances, the Intelsat VI recovery could have been effected with substantial benefits accruing to all parties.

\section{A NASA Group Task Force re-evaluation of its Shuttle program priorities and objectives after the Challenger loss concluded that in spite of the considerable need for spacecraft servicing, it does not serve NASA's interests to actively pursue the market using the Shuttle, for the primary reasons of safety and cost. This opens the door for the introduction of a dedicated OSS system designed along commercial lines.}

There is no question that OSS has the potential to be a commercially viable and profitable business. The economics however are complex and represent a major hurdle in the transition from OSS concept studies to development of an operational system. The US Department of Commerce forecasts a commercial space market in the billions 
of dollars. The challenge facing a potential OSS developer is to select a market and define a system architecture that will offer sufficient potential returns at an acceptable risk level. Another obstacle to be overcome is the implementation of a commercially viable OSS system when existing spacecraft are not designed to accommodate servicing. The possible solutions to this problem are: (a) to develop an initial system providing limited services to existing spacecraft; (b) to develop an OSS system in conjunction with a new generation of serviceable spacecraft; (c) to respond to any future political legislation, for example the introduction of a policy on satellite recovery or disposal which may result from the space debris problems.

Concern with regard to orbital crowding and space debris is mounting. The situation in both LEO and GEO locations is becoming critical, and the move towards Smallsats will only accelerate the problem. Resolution of this, though difficult to quantify on a purely economic level, could be a catalyst in bringing OSS into being.

The design, manufacture, launch and operation of space systems will always be a costly undertaking. The benefits associated with repair and refurbishment will therefore continue to be attractive. The implementation requires an economically or politically viable concept for servicing satellites. The balance may eventually be tilted in favour of OSS if spacecraft interfaces were standardized, or if international legislation were enacted to enforce or encourage the recovery or disposal of spacecraft. Assessment of the trends and the forces at work, it seems safe to state that space servicing will become a reality it is just a matter of time.

\section{SPACE ROBOTICS AND OSS}

\section{Overview}

OSS will require the next generation of autonomous, semi-autonomous or teleoperated robotics with advanced ground control - a natural progression from the manned robotics technology of the Space Shuttle (SRMS) and Space Station (MSS) programs. Indeed, looking to the future, since automated robots will be a key element in planetary exploration programs targeted for the next century, OSS development would appear to be to be a strategically astute policy for the space community. Participation in concept studies, technology demonstration programs, and development programs for satellite servicing can potentially facilitate the ongoing development of space robotics and its associated technologies. A strong case can be made that international collaboration is necessary in the evolution of OSS because of the anticipated high system implementation costs. It also promotes cooperation in the establishment of roles in a multinational effort that will produce global benefits.

\section{Technology Discussion}

The technologies associated with space robotics that are key to the development of an OSS system are discussed below.

Robotics, Tools \& Mechanical Interfaces 
In the broadest sense, unmanned spacecraft are themselves robots, but in this context the definition applies to manipulators, tools and devices for OSS tasks such as spacecraft capture, handling, berthing, end effector positioning, mechanical intervention, repairing, refurbishing, ORU changeout etc.

\section{Vision Systems \& Sensing}

Operational requirements for OSS include target identification, ranging, 3D mapping, multispectral sensing, lighting, photogrammetry. Much applicable work is being done in the area of hazardous waste remediation.

\section{Telefunction}

Telefunction refers to all aspects of the control of an advanced space system from the ground. It includes teleoperation, ground/space partitioning, predictive displays, data processing and much more.

\section{Autonomous Rendezvous and Docking}

This is really the combined application of many other technologies such as automated and remote controlled robotics, vision systems, mechanical interfaces, telefunction, power \& data transfer, communications.

\section{Communications}

Satellite/ground communications is a critical area for OSS due to the need for transmission of data for control of complex tasks. As stated, data bandwidth limitations and signal latency are key issues.

\section{CONCLUSIONS}

The level of space robotics activity within the global community supports the view that it is a critical technology, and will continue to be so as we move towards the exploration of our solar system and beyond. The U.S., Canada, Japan and Europe have at the same time independently and almost simultaneously identified on-orbit spacecraft servicing (OSS) as a promising endeavour, and one that provides an opportunity for application of this robotics technology. The major space industries are pursuing OSS concept studies, technology demonstrations and program definitions. It is widely accepted that these initiatives will lead to the establishment of multinational alliances in future OSS programs. Furthermore, it is proposed that OSS will be the first major commercial application of remote controlled and autonomous space robotics.

\section{REFERENCES:}

1. Satellite Services Systems Analysis Study - NASA Johnson Space Center Contract NAS 9-16121, Lockheed Missiles and Space Company, August 1982

2. Satellite Services Systems Analysis Study - NASA Johnson Space Center Contract NAS 9-16120, Grumman Aerospace Company, March 1983 
3. Satellite Servicing Mission Preliminary Cost Estimation Model - Science Applications International Corporation, NASA Johnson Space Center Contract NAS 9-17207, January 1987

4. Satellite Servicing Systems - Book of Issues, Engineering Directorate, Flight Projects Engineering Office, NASA Johnson Space Center, August 1987

5. Long-Term Orbital Lifetime Predictions - National Technical Information Service, 1990

6. Telerobotics, Automation, and Human Supervisory Control, Thomas B. Sheridan, MIT Press, (C) 1992 Massachussets Institute of Technology

7. NASA Report of the Group Task Force on Satellite Rescue and

Repair, September 1992 


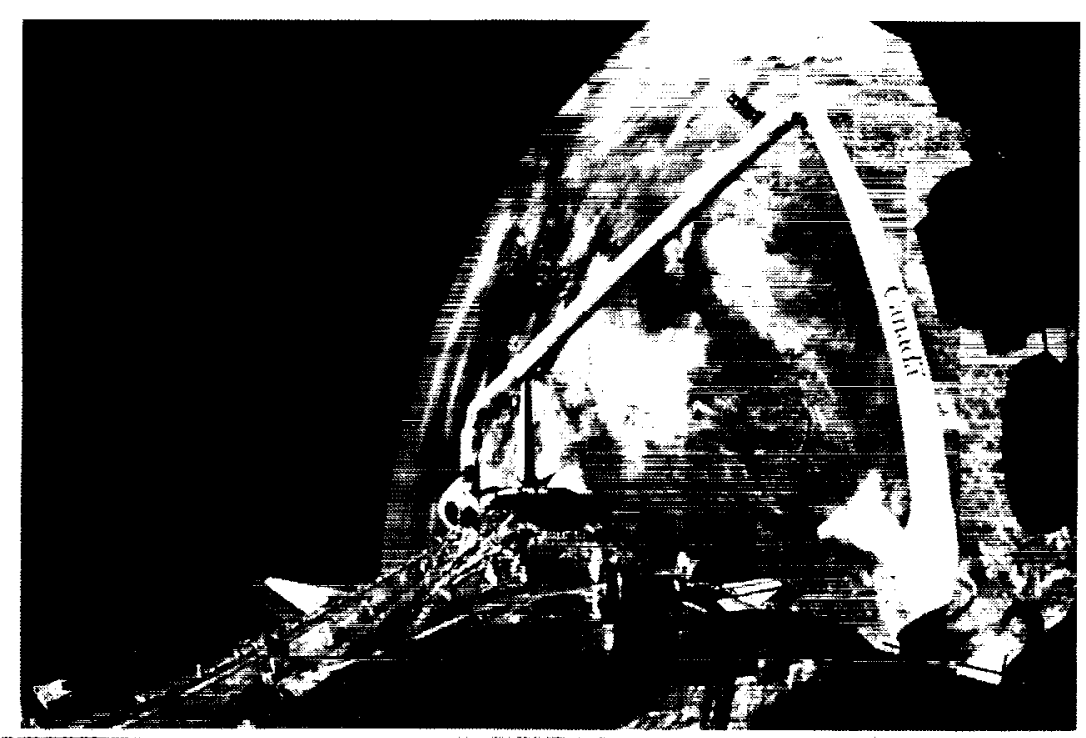

Figure 1. SRMS On-Orbit

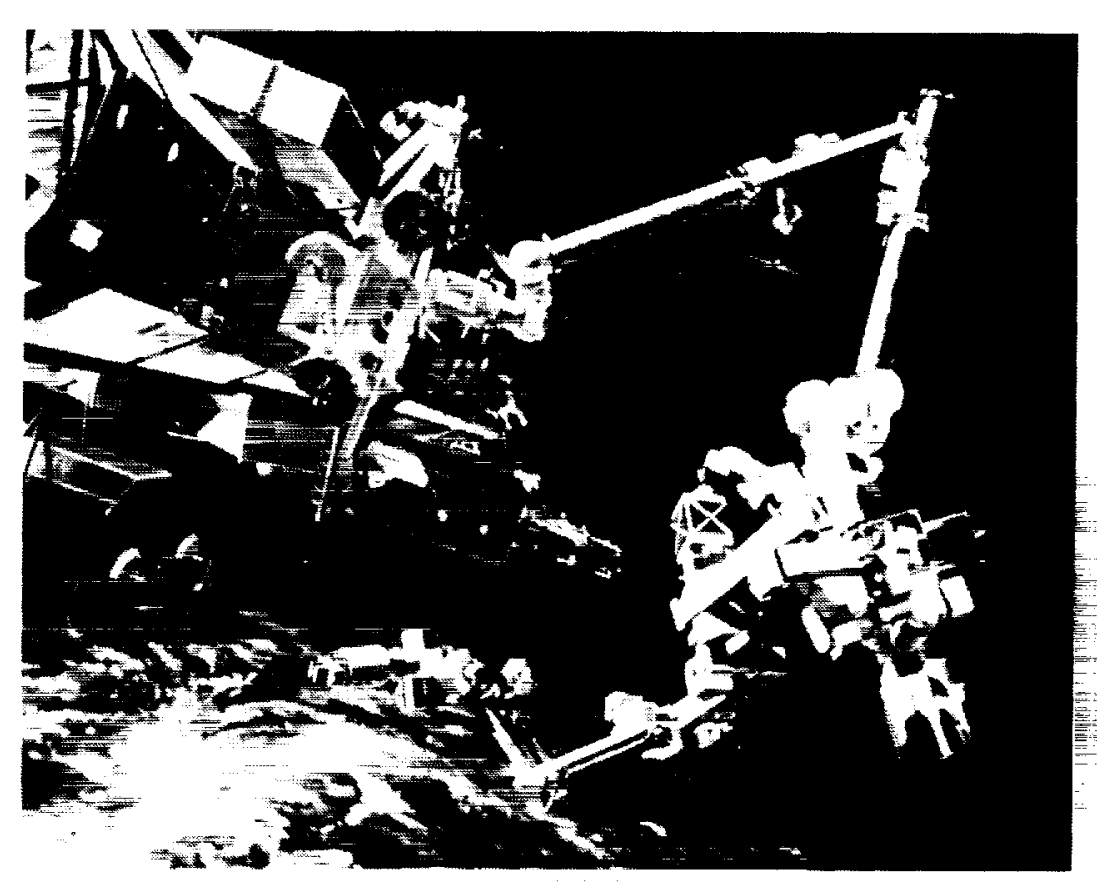

Figure 2. MSS Concept Illustration 